\title{
Acidificación urinaria basal en escolares normales
}

EDECIA OCHOA A. ", GILDA BORIE B. **, MIRIAM CORTES N. **

Una función importante del riñón es la de regular el equilibrio ácido-base de la sangre, lo que se logra variando la excreción diaria de $\mathrm{H}^{+}$por la orina.

En condiciones normales el organismo humano está sometido a una sobrecarga ácida importante, derivada especialmente de la metabolización de proteínas y lípidos que contienen grupos fosfatos y sulfatos. En el individuo sano esto lleva a la producción de orinas de acidez variable sin modificaciones importantes del $\mathrm{pH}$ sanguíneo. Sin embargo la función de acidificación urinaria puede alterarse por distintas causas llevando a patología importante.

La alteración de los parámetros de acidificación urinaria observada en niños de edad escolar con patología renal ${ }^{1}$ nos ha planteado la necesidad de determinar estos parámetros en niños sanos de edades comparables con igual técnica.

Se presentan los resultados obtenidos al determinar la excreción urinaria de hidrogeniones en escolares sanos con dieta libre.

Material Y Métódo. Se estudian 9 escolares norma'es cuyas edades fluctúan entre 6 y 14 años enviados la mayoría de ellos desde el Centro de Crecimiento y Desarrollo del Area Norte, al laboratorio de Exploración Renal del Hospital Roberto del Río.

Sin indicación de ayuno previo se toma muestra de sangre venosa, en forma anaeróbica, en jeringa heparinizada determinándose de inmediato $\mathrm{pH}$ sanguíneo, para esto se usó un $\mathrm{pH}$ metro Radiometer con microelectrodo a $38^{\circ} \mathrm{C}$ y se calcula $\mathrm{pCO}_{2}, \mathrm{HCO}_{3}^{-}$standard, $\mathrm{HCO}_{3}-$ actual y \footnotetext{
* Médico de Servicio de Nefrología, Hospital Roberto del Rio
y del Departamento de Mcdicina Experimental, Sede Norte. ** Químico Farmacéutico, Laboratorio de Exploración Renal, Hospital Roberto del Ríu.
}

exceso de base (B.E.) según el nomograma de Siggard-Andersen.

Se hizo recolección minutada de orina bajo vaselina por vaciamiento espontáneo por un período de aproximadamente 4 horas, en ella se determinó:

- pH en pHmetro Radiometer Modelo 27 con electrodos combinados.

- Acidez titulable: con hidróxido de sodio valorado, hasta $\mathrm{pH} \mathrm{7,4} \mathrm{en} \mathrm{pHmetro} \mathrm{Radiometer}$ modelo 27.

- Amonio por fotocolorimetría, método de nesslerización directa.

- $\mathrm{HCO}_{3}-$ en microgasómetro Natelson.

- Creatinina: por el método de Jaffé.

La velocidad de filtración glomerular se determinó por la depuración de creatinina verdadera según el método de Hare.

En todos estos niños la proteinuria realizada por técnica turbidimétrica con ácido sulfosalicílico fue negativa y la velocidad de filtración glomerular fue normal (Tabla I).

Resultados. Los pacientes fueron 7 hombres y 2 mujeres cuyas edades fluctuaron entre 6 y 14 años con superficies corporales que variaron entre 0.86 y $1,15 \mathrm{~m}^{2}$ siendo el promedio $1,05 \mathrm{~m}^{2}$. La velocidad de filtración glomerular promedio fue 119,4 con rango entre 104 y $138 \mathrm{ml} /$ minuto referido a la superficie corporal del adulto ( Tabla 1). El pH en sangre venosa promedio fue 7,38 con D.S. de 0.04 . La $\mathrm{pCO}_{2}$ promedio fue $36,7 \pm 4,9 \mathrm{~mm} \mathrm{Hg}$ y la bicarbonatemia 20,9 
TA B L A N 1

CARACTERISTICAS FISICAS Y VELOCIDAD DE FILTRACION GLOMERULAR DE ESCOLARES NORMALES

\begin{tabular}{|c|c|c|c|c|}
\hline$N^{o}$ & $\begin{array}{c}\text { Edad } \\
\text { (Años) }\end{array}$ & Sexo & $\begin{array}{l}\text { Superf. } \\
\text { Corporal } \\
\left(\mathrm{m}^{2}\right)\end{array}$ & $\begin{array}{c}V . F . G . \\
\left(\mathrm{cc} / \mathrm{min} / 1.73 \mathrm{~m}^{2}\right)\end{array}$ \\
\hline 1 & 9 & Masculino & 1.02 & 110.0 \\
\hline 2 & 6 & Masculino & 0.86 & 138.0 \\
\hline 3 & 9 & Masculino & 0.92 & 138.0 \\
\hline 4 & 14 & Masculino & 1.55 & 104.7 \\
\hline 5 & 6 & Masculino & 0.87 & 104.0 \\
\hline 6 & 11 & Femenino & 1.15 & 105.0 \\
\hline 7 & 9 & Femenino & 0.94 & 137.6 \\
\hline 8 & 8 & Masculino & 0.91 & 113.0 \\
\hline 9 & 13 & Masculino & 1.23 & 124.0 \\
\hline \multicolumn{3}{|c|}{$\bar{x} \pm D S$} & $1.05 \pm(0.11)$ & $119,4 \pm 16.0$ \\
\hline
\end{tabular}

$\pm 2,2 \mathrm{mEq} / \mathrm{lt}$. El exceso de base promedio fue $-2,7 \mathrm{mEq} / \mathrm{lt}$. con rango $-, 5,5$ y 0 . Los valores obtenidos se resumen en ta Tabla 2 .

El resultado de los parámetros de acidificación urinaria se presenta en la Tabla 3 . El pH en orina recién emitida varió entre 4.8 y 6.2 con promedio de 5.3.

La excreción urinaria neta de hidrogeniones $(\mathrm{AT}+\mathrm{NH} 4)-\mathrm{HCO}_{3}-$ fue promedio 53,8 $\mathrm{uEq} / \mathrm{min} / 1.73 \mathrm{~m}^{2}$ con rango entre 29,4 y 73,1 $\mathrm{uEq} / \mathrm{min} / 1,73 \mathrm{~m}^{2}$.

Comentarios. Existe un buen número de publicaciones que informan con respecto a la capacidad de acidificación de los niños, incluyendo la mayoría de ellos estudios funcionales completos $(2,3,4,5,6)$. Así pues se conocen los valores normales de la capacidad máxima de acidificación urinaria empleando pruebas de sobrecarga ácida, usando diferentes sustancias (6) y se tiene una idea bastante aproximada de la forma en que

T A B L A No 3

VALORES EN SANGRE VENOSA DE ESCOLARES NORMALES

\begin{tabular}{lccccc}
\hline$N^{o}$ & $p H$ & $\begin{array}{c}\mathrm{PCO}_{2} \\
(\mathrm{mmHg})\end{array}$ & $\begin{array}{c}\mathrm{HCO}_{3} \mathrm{st} \\
(\mathrm{mEq} \%)\end{array}$ & $\begin{array}{c}\mathrm{HCO}_{3} \text { Act } \\
(\mathrm{mEq} \%)\end{array}$ & $\begin{array}{c}\text { B. E. } \\
(\mathrm{mEq} \%)\end{array}$ \\
\hline 1 & 7.36 & 36.8 & 21.0 & 19.5 & -4.0 \\
2 & 7.30 & 47.8 & 22.0 & 23.0 & -3.0 \\
3 & 7.34 & 36.5 & 22.0 & 19.0 & -5.5 \\
4 & 7.42 & 30.5 & 20.0 & 19.0 & -3.0 \\
5 & 7.42 & 34.0 & 22.5 & 21.8 & -2.0 \\
6 & 7.40 & 38.5 & 24.2 & 23.5 & -5.5 \\
7 & 7.36 & 33.5 & 20.0 & 18.0 & 0 \\
8 & 7.41 & 39.0 & 24.5 & 24.0 & -1.5 \\
9 & 7.41 & 34.0 & 23.0 & 21.0 & $2.7 \pm 2.05$ \\
\hline
\end{tabular}

T A B L A No 2

VALORES EN ORINA DE ESCOLARES NORMALES

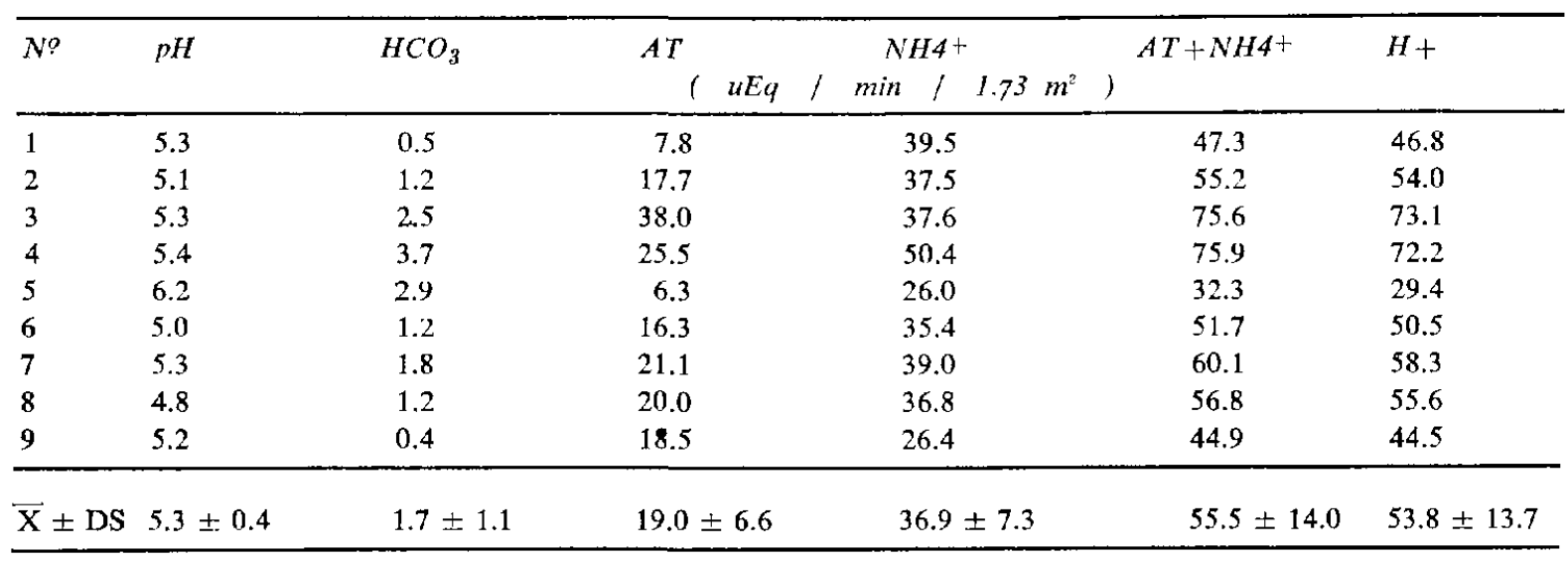


el riñón del niño (2) y del lactante menor maneja el bicarbonato (3). Eso sí, el método usado para determinar la capacidad máxima de reabsorción de bicarbonato ha sido impugnado porque produce expansión del espacio extracelular, situación que influye sobre la absorción de bicarbonato en el túbulo proximal. $(7,8)$.

Nuestra intención al efectuar este trabajo ha sido solamente tener valores de excreción urinaria de hidrogeniones en niños sin patología renal para compararla con la obtenida en pacientes con acidosis tubular en edad escolar.

Los resultados obtenidos con nuestras técnicas no difieren mayormente de otras publicaciones.

Cabe destacar eso sí que los niños de nuestra serie presentan valores más bajos de los parámetros en sangre que otras series publicadas (2, 3 ). Pensamos que esto puede estar en relación a períodos más o menos prolongados de ayuno; pese a la indicación de asistir al laboratorio habiendo desayunado, pudimos comprobar en un par de niños que la última comida recibida había sido más de 12 horas antes de la toma de muestras; en estos niños se comprobó acidosis metabólica con excesos de base alrededor de $-8 \mathrm{mEq} /$ 1t. No se incluyen en este estudio.

\section{BiBLIOGRAFÍA}

1.-Ochoa, E., Borie, G., Cortés, M. "Acidosis tubular renal. Presentación de 2 casos clínicos". Rev. Chilena de Pediatría.
2.-Edelmann, C. M., Boichis, C. M. Rodríguez-Soriano, J., Stark, H. "Renal response of children to acute ammonium chloride acidosis". Pediat. Res. 1: $452,1967$.

3.-Edelmann, C. M., Rodríguez-Soriano, J., Boichis, H., Gruskin, A., Acosta, M. "Renal bicarbonate reabsorption and Hydrogen ion excretion in normal infants". J. of Clin. Invest. 46: 1306, 1967.

4.--Rodríguez-Soriano, J., Boichis, H., Edelmann, C. $M$. "Bicarbonate reabsorption and bydrogen ion excretion in children with renal tubular acidosis". J. of Pediat. 71: 802, 1967.

5.-Broyer, M., Proesmans, W., Royer, P. "La tritration des bicarbonates chez l'enfant normal et au cours de diverses néphropathies: Rev. franc. Etud. Clin. Biol. 14: 556, 1969.

6.- Toursel, F. "Exploration de la fonction renale d'acidification par les épreuves de surcharge acide chez l'enfant". Rev. de Pediat. 8: 115, 1972.

7.- Rodriguez-Soriano, J. "The renal regulation of acidbase balance and the disturbances noted in renal tubular acidosis". Pediat. Clin. of North America 18: $529,1971$.

8.-Kurtzman, N. A. "Regulation of renal bicarbonate reabsorption by extracellular volume". J. of Clin. Invest. 49: 586, 1970.

Se agradece a la Sra. Ilse Oberhauser, Sra. Olga Basulto, Srta. Cecilia Silva y Sra. Yolanda Arriagada del Departamento de Medicina Experimental, Area Norte, por la valiosa cooperación prestada, y al Dr. Alfredo Avendaño, quien envió la mayoría de los pacientes a nuestro Laboratorio. 